\title{
ACCURATE NUMERICAL SOLUTIONS TO STATIONARY FREE SURFACE PROBLEMS FROM CAPILLARITY
}

\author{
C.-I. Gheorghiu
}

T. Popoviciu Institute of Numerical Analysis, Cluj-Napoca, Romania

The paper considers two static problems from capillarity. The first one consists in the determination of the surface of a liquid in a capillary tube and the second in the computation of the shape of a sessile or pendent drop of liquid of a given volume, both configurations being considered in the gravitational field. From the mathematical point of view both problems are nonlinear two-point boundary value problems which include in their formulations additional geometrical unknowns, i.e., the so-called free boundary value problems. Computationally they are laborious problems since they involve non-normal Jacobian matrices. The resulting numerical difficulties are considerably reduced by treating these problems by a collocation method in conjunction with the continuation with respect to the parameters. The method is implemented by the MATLAB code $b v p 4 c$. The numerical evaluations of the free surfaces are in reasonable accordance with asymptotic estimations.

Рассматриваются две статические задачи теории капиллярности. Первая заключается в определении поверхности жидкости в капилляре, вторая - в вычислении формы неподвижной или висячей капли жидкости заданного объема. Обе конфигурации рассматриваются в гравитационном поле. С математической точки зрения обе задачи представляют собой нелинейные двухточечные краевые задачи, которые в своей постановке содержат дополнительные геометрические неизвестные, т. е. так называемые краевые задачи со свободными границами. В вычислительном смысле это очень трудоемкие задачи, так как они содержат ненормальные матрицы Якоби. Вычислительные трудности существенно упрощаются при решении этих задач путем объединения метода коллокаций с методом продолжения по параметрам. Метод реализован в программе MATLAB с помощью $p v p 4 c$. Численные оценки для свободных поверхностей находятся в приемлемом согласии с асимптотическими оценками.

PACS: 02.60.Lj; 47.55.nb

\section{INTRODUCTION}

In many cases, problems in viscous fluid mechanics can be described by boundary value problems for partial differential equations. In some cases, by using the geometrical symmetries of the configurations such problems can be reduced to two-point boundary value problems. When there are additional geometrical unknowns we speak of free boundary value problems (FB for short). More specifically, these geometrical unknowns can be some values of the unknowns on subsets of boundary points or even the coordinates of the boundary points themselves. In this paper we solve numerically two classes of static capillary FBs. These problems are defined as FBs on which we must take into account capillary forces, i.e., forces due to the intermolecular attractions which have a nonvanishing resultant at the boundary of the liquid. 
The first one is to determine the liquid-air interface (surface) whose mean curvature is proportional to its height above the reference plane, and which makes with a container bounding wall a prescribed constant angle. The second one is to find the shape of a sessile or pendent liquid drop of a given volume which rests in the gravitational field. The actual computations were carried out for symmetric cases. The equations of these problems, as well as the analytic properties of the solutions (existence, uniqueness and asymptotic behavior for Bond number approaching 0), were established in the monograph of R. Finn [1]. Both problems lead to nonlinear second-order two-point free boundary value problems; i.e., the values of the solution at some boundary points are unknown but some values of the derivatives are supplied in order to close the problem. Their solutions depend on the nondimensional Bond number (Bo for short) which is a measure of the «size» of the configuration; i.e., it is the ratio of gravitational forces to the capillary (superficial) forces. The main computational difficulty with these problems consists in the fact that they involve non-normal Jacobian matrices. A test problem with a Jacobian matrix in close form is considered in order to underline these aspects.

The problems are solved numerically making use of a collocation type method, based on cubic interpolation, and introduced in the paper of Kierzenka and Shampine [3]. It is implemented by the MATLAB code $b v p 4 c$ and provides a smooth solution continuous along with its first derivative. In contrast with well-known shooting, this solution is approximated on the whole interval and the boundary conditions are taken into account at all times. As the resulting nonlinear system of algebraic equations is solved iteratively the question of coming up with sufficiently accurate initial guess of unknowns is particularly discussed.

\section{A TEST PROBLEM}

In their paper [4], Miele, Aggarwal and Tietze consider the following two-point boundary value problem introduced by Troesch:

$$
x_{t}=y, \quad y_{t}=k \sinh (k x), \quad 0<t<1, \quad x(0)=0, x(1)=1 .
$$

The Jacobian matrix

$$
J=\left(\begin{array}{cc}
0 & k^{2} \cosh (k x) \\
1 & 0
\end{array}\right)
$$

is characterized by the eigenvalues $\lambda= \pm k \sqrt{\cosh (k x)}$, which at the endpoints become $\lambda(0)= \pm k$ and $\lambda(1)= \pm k \sqrt{\cosh (k)}$.

For relatively low values of $k$, i.e., $0<k \leqslant 4$, the above eigenvalues remain relatively small and the problem can be solved employing the usual finite difference or the shooting methods. On the other hand, for relatively large values of $k$, i.e., $5 \leqslant k \leqslant 10$, the abovequoted authors make use of a special multipoint boundary-value technique in order to solve the problem. They observe that the problem is characterized by the first integral

$$
z:=\cosh (k x)-y^{2} / 2=\text { const }
$$

and use that as a «tool» to check the accuracy of the numerical solution. In our numerical experiments performed on a Pentium IV, 3.0G, PC, the constancy of $z$ is verified to a maximal 
error equal to $4.082997 e-06$ for $k=5$, to an error which equals $7.651421 e-03$ for $k=10$ and to an error equal to $6.602193 e+00$ corresponding to $k=15$. Our numerical results are with one significant figure worse than those reported in [4, Table II, p. 126], for $k=5$ and $k=10$, respectively. For $k$ larger than 16 the accuracy in the conservation of $z$ is lost rapidly. In spite of this fact, even at these values of $k$ our numerical solutions behave correctly in the left neighborhood of $t=1$. They show a boundary layer type behavior in this region.

It is worth noting at this point that we used essentially the technique of continuation with respect to parameter $k$ in order to handle the problem for $k \geqslant 15$. Without this technique for such values of $k$ the collocation equations become unsolvable due to singular Jacobian.

Remark 1. In fact the above Jacobian matrix $J$ is non-normal, i.e., $J^{*} J-J J^{*} \neq 0$, where $*$ stands for the conjugate transpose of a matrix (see Trefethen [5, p. 187]). The pseudospectrum of the matrix $J$, at $t=1$, i.e., the spectrum of the randomly perturbed matrix with an arbitrary small quantity, is depicted in Fig. 1. In our previous paper [2] we introduced a scalar measure of non-normality. It reads $H(J):=\sqrt{\varepsilon\left(J^{*} J-J J^{*}\right)} / \varepsilon(J)$ and we noticed that $0 \leqslant H(J) \leqslant \sqrt[4]{2}, 0$ being attained just in case of normal (symmetric) matrices. On this scale our matrix $J$

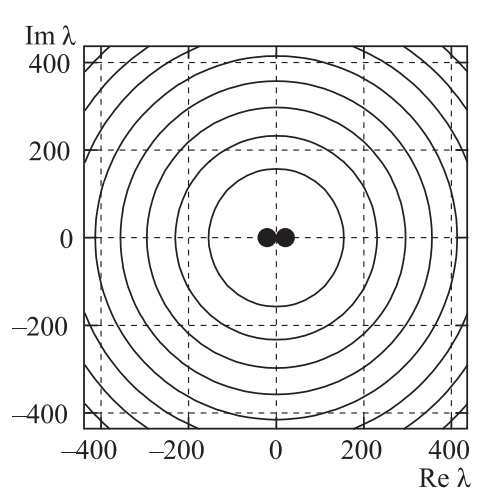

Fig. 1. The pseudospectrum of matrix $J$ for $k=4$ is situated extremely close to the upper bound. This scalar measure, as well as the pseudospectrum, leads us to the conclusion that the matrix $J$ is a genuine non-normal one.

\section{THE LIQUID-GAS INTERFACE IN A CAPILLARY TUBE}

In this section we consider static capillary liquid-gas interfaces of liquids partly filling a vessel. More exactly, we examine the behavior of capillary surfaces in a tube with circular section of radius $R=a$. We assume the following system of two coupled nonlinear differential equations and boundary conditions (see [1, p. 11]):

$$
\begin{aligned}
& \operatorname{div}(T u)=\operatorname{Bo} \cdot u, \text { in } \Omega, \\
& \nu \cdot T u=\beta, \text { on } \Sigma:=\partial \Omega,
\end{aligned}
$$

where $u(x, y)$ denotes the height over the wetted section $\Omega \subset \mathbb{R}^{2}, T(u):=\frac{\nabla u}{\sqrt{1+|\nabla u|^{2}}}$, $\beta$ is known and $\nu$ is the outward normal to $\Sigma$.

Due to the symmetry with respect to the axis of the tube, on the half of the axial section, i.e., $0 \leqslant r \leqslant 1, r:=R / a$, the problem reads

$$
\begin{gathered}
\left(\frac{r u_{r}}{\sqrt{1+u_{r}^{2}}}\right)_{r}=\mathrm{Bo} \cdot r u, \quad 0<r<1, \\
u(0)=p_{1}, \quad u_{r}(0)=0, \quad u(1)=p_{2}, \quad \cos (\delta)=\beta, \quad r=1,
\end{gathered}
$$


where Bo $:=\rho g a^{2} / \sigma, \rho$ and $\sigma$ being respectively the density and the surface tension of the liquid and $g$ is the usual gravitational acceleration. The parameters $p_{1}$ and $p_{2}$ are unknown and signify the heights of the liquid on the axis of symmetry and on the wall of the tube, respectively. The boundary condition $u_{r}(0)=0$ appears due to the symmetry and $\delta$ is the angle between the liquid-gas interface $u(r)$ and the wall of the tube (see Fig. 2). The problem is effectively integrated as a first-order differential system, i.e.,

$$
\begin{gathered}
u_{r}=v, \quad v_{r}=-\frac{1}{r}\left(v+v^{3}\right)+\mathrm{Bo} \cdot u\left(v+v^{3}\right)^{3 / 2}, \quad 0<r<1, \\
u(0)=p_{1}, \quad u(1)=p_{2}, \quad v(0)=0, \quad \frac{v(1)}{\sqrt{1+v^{2}(1)}}=\beta .
\end{gathered}
$$
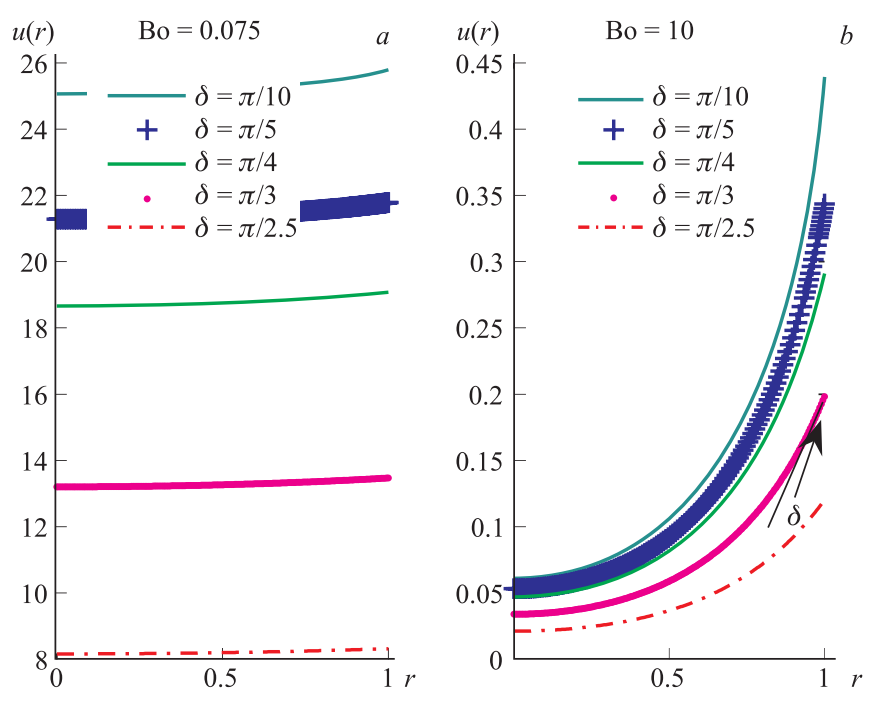

Fig. 2. The liquid-gas interface

The second differential equation in (5) is singular at $r=0$, but the singular coefficient arises from the coordinate system and we expect a smooth solution in the neighborhood of this point. We deal with this singularity using the technique suggested in Example 6 from [3, p. 18-19]. It means that we let $r \rightarrow 0$ in this equation and obtain the value that must be used for evaluating the differential equation when $r=0$.

The existence of symmetric solutions with prescribed angle $\delta$ has been proved and the uniqueness theorem shows that the symmetric solutions are the only ones (see [1] and the paper quoted there). For Bo $\rightarrow 0$, in the same monograph, the Laplace formula, which estimates $p_{1}$, and estimations for the parameter $p_{2}$ are also available. These estimations were fairly useful in guessing the initial shape of the unknown $u(r)$.

For $\mathrm{Bo}=0.075$ and $\mathrm{Bo}=10$, the dependence of $u(r)$ on the angle $\delta$ is depicted in Fig. 2 .

Remark 2. From computational point of view is suggestive to notice that the code bvp $4 c$ works such that the maximal residual belongs to the range $4.563 e-007$ and $7.354 e-004$. 


\section{THE SESSILE AND PENDENT DROPS}

We consider a liquid drop of volume $V$ resting on a horizontal plane (surface) in a vertical gravity field and restrict our attention to the case of the contact angle $\delta$ such that $0<\delta \leqslant \pi / 2$ (see Fig. 3). The case $\delta>\pi / 2$ reduces to that one under the transformation $u \rightarrow-u$. Supposing that the drop is symmetric, the equations for the surface of the drop are

$$
\begin{gathered}
u_{r}=v, \quad v_{r}=-\frac{1}{r}\left(v+v^{3}\right)+(\mathrm{Bo} \cdot u+\lambda)\left(v+v^{3}\right)^{3 / 2}, \quad 0<r<1, \\
u(0)=p, \quad v(0)=0, \quad u(1)=0, \quad \frac{v(1)}{\sqrt{1+v^{2}(1)}}=\beta
\end{gathered}
$$

where $\lambda$ is a Lagrangian multiplier corresponding to the volume constraint. It is shown (see [1, p. 59-60]) that if $\delta=$ const, then all equilibrium surfaces are symmetric and there is a one-to-one correspondence between capillary surfaces furnished by (4) and sessile drops implied by (6). Our numerical results are reported in Fig. 3.

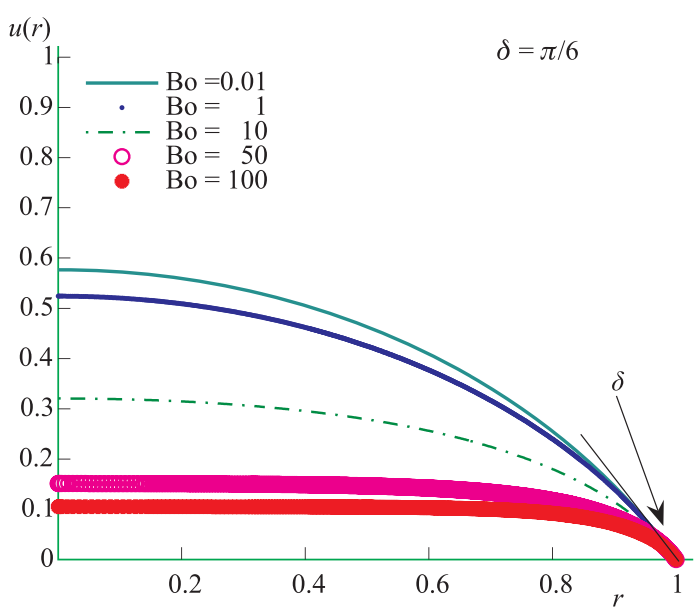

Fig. 3. The shape of the sessile drops

Remark 3. An upper bound for the existence of the solution with respect to Bo can be obtained. The principle of virtual work applied to the energy functional of the drop of volume $V$, yields a slightly modified form of (3), namely

$$
\begin{gathered}
\operatorname{div}(T u)=\mathrm{Bo} \cdot u+\lambda, \text { in } \Omega, \\
\nu \cdot T u=\beta, \text { on } \Sigma:=\partial \Omega,
\end{gathered}
$$

where $\lambda$ is associated with the volume restriction. The volume $V$ of the drop can be expressed explicitly in terms of the boundary data and this property holds for general section $\Omega$. We integrate the first equation in (3) and introduce the boundary condition. Thus, the volume is expressed as

$$
V=\frac{1}{\mathrm{Bo}}(\Sigma \cos \delta-\lambda \Omega)
$$


where the symbols $\Sigma$ and $\Omega$ denote respectively the measures of these sets. For symmetric solutions, (8) becomes

$$
V=\frac{\pi}{\mathrm{Bo}}(2 \cos \delta-\lambda)
$$

Taking into account the boundedness of $\cos \delta$ the last equality implies

$$
\mathrm{Bo} \leqslant \frac{2-\lambda}{2 \int_{0}^{1} u(r) d r}
$$

As the Lagrangian multiplier $\lambda$ is delivered by bvp $4 c$ this upper bound for Bo can be verified numerically. The results of our numerical experiments show that the quantity $\frac{2-\lambda}{2 \int_{0}^{1} u(r) d r}$ overestimates the $\mathrm{B}$ number for $\mathrm{Bo} \leqslant 10$ and unfortunately underestimates that for $\mathrm{Bo}>10$. It remains an open problem to explain why for large values of Bo this inequality is not fulfilled.

\section{CONCLUSIONS}

The numerical experiments reported in this paper, as well as some other which are in progress and refers to FB problems from laminar boundary layer theory, entitle us to think that the main difficulty with this type of problems consists in the high non-normality of their Jacobian matrices. The collocation method implemented by $b v p 4 c$, due to its global character, overcomes to some extent these difficulties. As a general remark we have to observe that the problems corresponding to microgravity conditions, i.e., Bond number closed to zero, are easier to be solved and the results are much more accurate than those obtained when this number is increased.

\section{REFERENCES}

1. Finn R. Capillary Free Surfaces. Preprint No. 7/1981. Bonn: Inst. für Angewante Mathematik Univ., 1981.

2. Gheorghiu C.-I. // Mathematica. Studia Univ. «Babes-Bolyai». 2005. V. L. P. 61.

3. Kierzenka J., Shampine L.F. // ACM Trans. Math. Softw. 2001. V.27. P. 299.

4. Miele A., Aggarwal A. K., Tietze J. L. // J. Comp. Phys. 1974. V.15. P. 117.

5. Trefethen L. N., Bau III D. Numerical Linear Algebra. SIAM, 1997. 